1

2

3

4

$5 \quad{ }^{1}$ Center for Mind/Brain Sciences, University of Trento, Rovereto, Italy

6

7 Corresponding authors:

8

9 Davide Potrich, Giorgio Vallortigara

10 Piazza Manifattura,1 38068 Rovereto

11

12

13

14

15 Orcid IDs:

16

17 Mirko Zanon: 0000-0003-4062-1496

18 Giorgio Vallortigara: 0000-0001-8192-9062

\section{Archerfish number discrimination}

Davide Potrich ${ }^{1}$, Mirko Zanon ${ }^{1}$, Giorgio Vallortigara ${ }^{1}$

+390464808736

davide.potrich@unitn.it; giorgio.vallortigara@unitn.it
Davide Potrich: 0000-0003-0928-628X 
ABSTRACT

Debates have arisen as to whether non-human animals actually can learn astract nonsymbolic numerousness or whether they always rely on some continuous physical aspect of the stimuli covarying with number. Here we investigated archerfish (Toxotes jaculatrix) non-symbolic numerical discrimination with accurate control for co-varying continuous physical stimulus attributes. Archerfish were trained to select one of two groups of black dots (Exp. 1: 3 vs. 6 elements; Exp. 2: 2 vs. 3 elements); these were controlled for several combinations of physical variables (elements' size, overall area, overall perimeter, density and sparsity), ensuring that only numerical information was available. Generalization tests with novel numerical comparisons (2 vs. 3, 5 vs. 8 and 6 vs. 9 in Exp. 1; 3 vs. 4, 3 vs. 6 in Exp. 2) revealed choice for the largest or smallest numerical group according to the relative number that was rewarded at training. None of the continuous physical variables, including spatial frequency, were affecting archerfish performance. Results provide evidence of the spontaneous use of abstract relative numerical information in archerfish for both small and large numbers.

KEYWORDS: number, numerical cognition, number discrimination, numerical rule, 


\section{INTRODUCTION}

40 Non-symbolic numerical estimation is an important and well-studied cognitive ability that

41 allows humans and other animals to interact successfully with their surroundings. The

42 development of a "sense of number" is associated with fundamental biological needs

43 that in many ecological contexts allow animals to estimate how many companions or

44 enemies are around, or how much food is present in different patches - all important

45 information to maximize fitness and reproductive success in the wild [1].

Typically, in order to assess numerical abilities animals are requested to

47 discriminate between sets of visual stimuli differing in numerosity (review in [2]). This

48 can be done using spontaneous attractive natural stimuli such as food or social

49 companion, taking advantage of the animals' natural and spontaneous tendency in

50 some ecological contexts to "go for more". Alternatively, operant conditioning

51 procedures can be used that associate a particular set of stimuli with a reward.

52 Extensive evidence supports the use of numerical information in non-human primates

53 (e.g., [3-7]), as well as in other mammals (e.g., [8-13]), in birds (e.g., [14-19]), in

54 amphibians (e.g., [20,21]), in reptiles (e.g., [22,23]), in fish (e.g., [24-26]) and in

55 arthropods (e.g., [27-30]) (see for general reviews in vertebrates [1,31,32]).

Numerical discrimination seems to be supported by an "Approximate Number

57 System" (ANS, [33,34]), which discriminative accuracy is ratio-dependent in accordance

58 to Weber's law (as the ratio between two numerosity increases, the discrimination gets

59 more difficult). Besides the ANS, an attentional working memory-based system has

60 been claimed for by some authors as providing precise representation of small numbers 
61 (up to 3-4), the so-called "Object Tracking System" (OTS; [35]), though its generality for

62 non-human animals is debated (discussion in $[31,36]$ ).

Studies investigating the neural basis of number representation revealed

64

65

66

67 selectivity of response of neurons in some areas of the brain such as the parietal and prefrontal cortex in humans $[37,38]$ and in monkeys $[39,40]$, the nidopallium caudolaterale in crows $[15,41]$ and the most caudal dorsal-central part of the pallium in zebrafish [42,43], suggesting that common selective pressures led to convergent evolution of numerical representation in different species $[44,45]$.

However, one issue in all these experiments is that animals are dealing with sets of physical elements, and thus numerical information is intrinsically melted with other non-numerical properties of the stimulus, such as the area, the density or the spatial frequency or the elements' arrangement [46]. Recently, some debates have arisen concerning whether bees use abstract numerical information or rather rely on sensory properties of the stimulus for discrimination $[47,48]$.

Taking advantage of the fact that we recently developed a sophisticated script for the automatic generation of visual stimuli that can allow proper randomization and control of continuous physical variables in number sense experiments [49], we decided to perform some very precisely controlled experiments to check whether fish do use number as abstract property.

We selected archerfish (Toxotes jaculatrix) for our study. These fish are wellknown for their particular hunting strategy, which consists of spitting at preys above the water surface with a precise jet of water thrown with the mouth. This attacking repertoire makes it very easy to train them to hit targets using operant conditioning (see e.g., [50]). 
Still, to date, no studies in archerfish have explicitly investigated abstract numerical abilities. Leibovich-Raveh et al. [51] and colleagues showed that when archerfish make magnitude-related decisions, their choice is influenced by the non-numerical variables

87 that positively correlate with numerosity; for instance, when exposed to two groups of dots differing in number and continuous physical information, archerfish spontaneously selected the group containing the larger non-numerical magnitudes and smaller numerosity, switching to the larger numerical set when positively correlated with all the non-numerical magnitudes.

Related to magnitude discrimination, archerfish also proved to be able to associate different geometric shapes with different food quantities [52]; this would support the existence of a system dealing with magnitudes, although a specific role of numerical information remains unclear. small and a large numerosity (Exp. 1:3 vs. 6 elements) or small numerosities only (Exp. 2: 2 vs. 3 elements). After reaching a learning criterion, archerfish were tested with novel numerical comparison (2 vs. 3, 5 vs. 8 and 6 vs. 9 in Exp 1; 3 vs. 4, 3 vs. 6 in Exp judgement (select the "largest" or "smallest" group) or on an absolute judgment (select a specific number of item). All of the different continuous physical variables such as radius, total area, total perimeter, convex hull and inter-distance were carefully controlled for and alternately balanced across trials, ensuring that the animals could not rely on them to perform their judgment (Figure 1). Furthermore, a statistical analysis was run for a posteriori evaluation of whether any of these variables influence the 

evaluation.

Figure 1. Schematic representation of the non-numerical physical controls applied to 113 the stimuli in each session. 
RESULTS

117 EXPERIMENT 1

118 Eight archerfish were trained to discriminate between two groups of black dots in a 3 vs.

1196 numerical comparison; four fish were trained to select the number 3, while the other 120 four were rewarded with the number 6 . No difference has been found in the number of

121 trials needed to reach the learning criterion between the group trained with 3 elements 122 (mean $\pm \mathrm{SEM}=451.25 \pm 106.77$ ) and the group trained with 6 elements (mean $\pm S E M=$ 413.25 \pm 73.14 ) (Independent Samples t-Test: $\mathrm{t}(6)=0.294, p=0.779)$.

124 Once the learning criterion was reached, all the fish performed three different tests. represented numerosity as relative or absolute. Fish trained to select the smallest

127 number 3 at training (i.e. the smallest set in the 3 vs. 6) were presented at test with a novel discrimination 2 vs.3, while fish trained to select the number 6 at training (i.e., largest set in the 3 vs. 6) were tested with a 6 vs. 9 condition. The use of "relative" information (go for the smallest or largest) should lead the fish to choose the novel numerosity at test, while the use of "absolute" information would reflect in the choice of the stimulus with the same number of elements as at training.

TEST 2: The second test aimed to clarify the role of the incorrect (i.e., unrewarded) training stimulus and its relevance for the fish. When fish are trained to select the numerosity 3 , thus avoiding number 6 , once presented with the new

136 comparison 6 vs. 9 (or vice versa 2 vs. 3, if trained to select 6), do they choose the 137 group according to the relative information even if it coincides with the absolute 138 numerosity to avoid at training? 
TEST 3: In the last test, fish behaviour was observed in a comparison involving novel numerosities never experienced during the training, i.e., 5 vs. 8. This allowed observing whether zebrafish applied a relative representation (go for the "smallest" or "largest"), or if the choice was at the chance level, since no absolute numerical information experienced at training was present here.

Results at tests for Experiment 1 are reported in Figure 2. Choices for the relative numerosity were analyzed using a generalized linear mixed model fit by maximum

146 likelihood (Laplace Approximation), binomial GLMM with a logit link in R. Four fixed 147 effects (type of Training -3 or 6 dots-; type of Test -2 vs. 3, 5 vs. 8 and 6 vs. 9-; type of 148 geometrical control -radius fixed, overall area controlled, overall perimeter controlled-; 149 type of spatial disposition control -inter-distance controlled; convex-hull controlled-) and 150 one random intercept effect (fish) were considered. Analysis on the random effect 151 showed not to affect the model and no significant differences were found between 152 effects of groups, nor group interactions (comparisons between different models 153 considering various effects and interactions reported always $p>0.05$, suggesting to 154 adopt the simplest model described by the only choice with no contribution from any 155 effects). Only a trend for the contribution of the type of geometrical control was 156 observed, driven by a non-significant difference between the "radius fixed" and "overall 157 area controlled" conditions (post-hoc non-parametric tests adjusted with Tukey method: $158 p=0.063)$; within this trend, every single condition was statistically significant by chance 159 level in the direction of the relative choice ("radius fixed": probability of success = $16071.18 \%, p<0.001$; "overall area controlled": probability of success $=81.25 \%, p<0.001$; 161 "overall perimeter controlled": probability of success $=73.61 \%, p<0.001)$. 


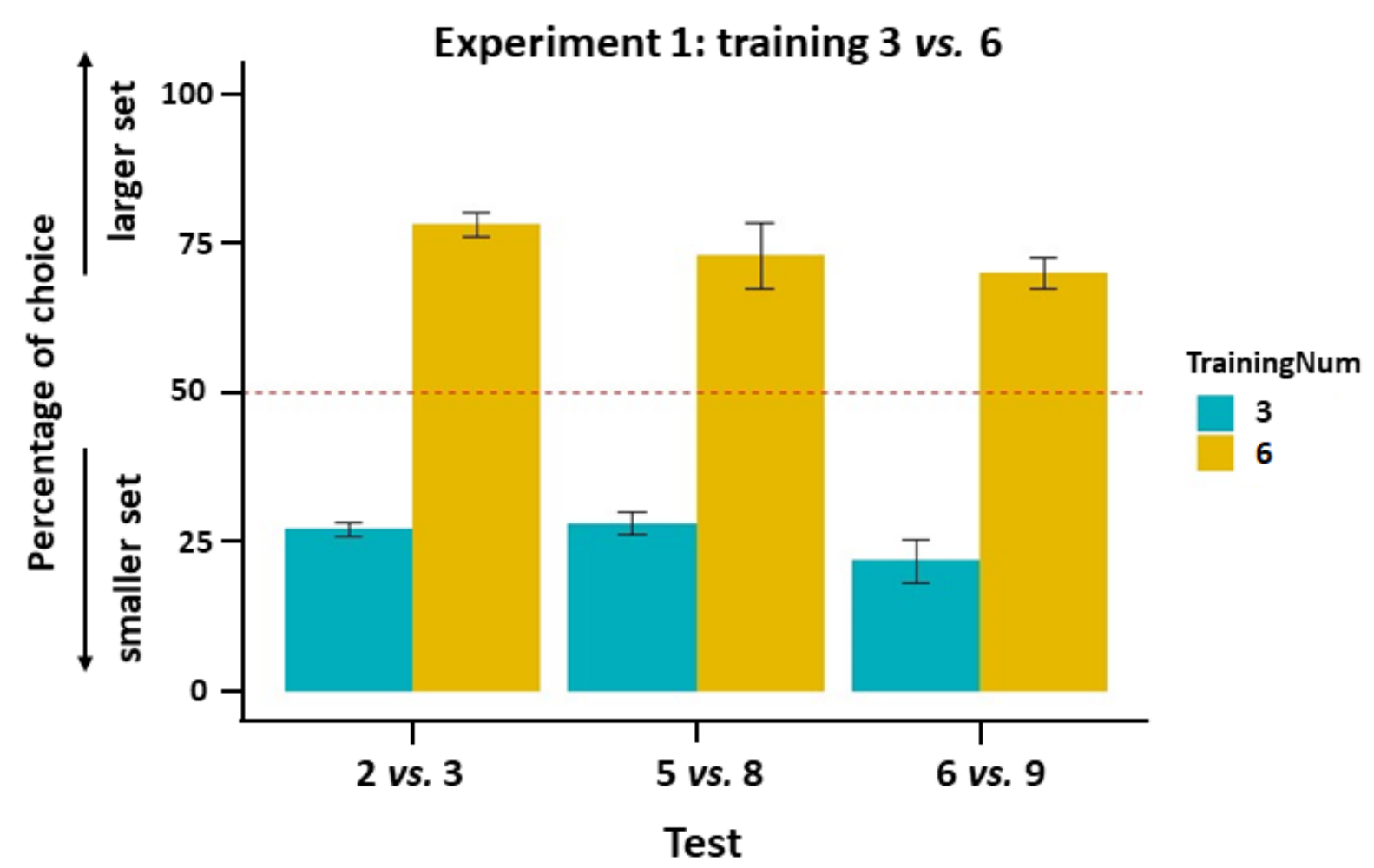

Figure 2. Percentage of choice for the larger/smaller set (mean \pm SEM) displayed by fish in the novel comparison tests for the groups trained to select the smaller (3) or larger (6) set.

Overall, considering the previous discussion, a binomial test shrinking all the data together was performed to investigate the final findings: fish showed a strong significant preference for the relative numerosity (probability of success: $74.3 \%, p<0.001$ ).

The result obtained in Experiment 1 showed that archerfish, when trained to select one of two simultaneously displayed groups of dots with different numerosities (i.e., 3 vs. 6 dots), use a relative numerical rule to perform novel numerical comparisons. These results confirm findings in other fish species such as angelfish [55] and guppy [56] but they are different from those obtained in bees which showed instead a preference for the absolute number [57]. An important difference between fish and bees studies is related to the numerical comparison used: respectively large numbers $(>4$ 
177 elements) for fish and small numbers ( $\leq 4$ elements) with bees. This might engage 178 different systems (see Introduction) thus explaining the discrepancy. The training discrimination used here in Experiment 1 involved two numbers (3 vs. 6) that belong one to the hypothesized "small" and the other to "large" systems, respectively. This is different than in previous fish studies which involved only large numerosities; thus, it remains to be tested how fish would deal when trained with small numerosities only. In principle, the presence of a large number in the comparison in Exp. 1 may be enough to

184 lead the archerfish to follow a relative rule. If trained with a numerical discrimination 185 involving only small numbers, would the animals still use a relative numerosity judgement or would they turn to absolute judgement? This was tested in Experiment 2.

\section{EXPERIMENT 2}

189 Four subjects were trained to select the largest number in a 2 vs. 3 comparison (i.e., the involving a comparison between the previously trained numerosity (3) and a novel 192 numerosity (4 or 6).

All fish reached the learning criterion, showing an ability to discriminate between

194 the two numbers (trials to criterion $\pm S E M=506.5 \pm 97.8$ ). Results at test are reported in 195 Figure 3. A GLMM model with three fixed effects (type of test -3 vs. 4 and 3 vs. 6-; type 196 of geometrical control -radius fixed, overall area controlled, overall perimeter controlled-; 197 type of spatial disposition control -inter-distance controlled; convex-hull controlled-) and 198 one random intercept effect (fish) showed no random effect of fish, neither significant 199 differences between groups or groups' interactions (Chi-Square tests between all 
200 different models with different effects and interactions report always $p>0.05$, suggesting

201 to adopt the simplest model based on the only fish choice -for the relative or absolute

202 number- and no effects of controls).

203 An Exact binomial test considering a merge of the data showed a highly 204 significant preference for the relative number (probability of success: $69.79 \%, p<$ $2050.001)$

In Experiment 2, archerfish showed to be able to discriminate between two 207 different numerical groups of dots within the small numerical range. At test, fish 208 preferred the novel numerosity to the familiar 3 items, in both 3 vs. 4 and 3 vs. 6 209 comparisons, confirming the use of a relative rather than absolute numerical rule. This 210 evidence does not match with findings in bees, tested in the same numerical conditions, 211 suggesting that the spontaneous engagement of relative/absolute rule to extract 212 numerical information may be guided by different ecological pressures experienced by 213 different species in their phylogenetic history. The spontaneous use of relative rules 214 suggests that among fish, it is more important to learn a general rule that is applicable 215 to novel comparisons. It cannot be excluded that this strategy is adopted because it 216 could be less demanding as to memory load than an absolute judgement strategy. 


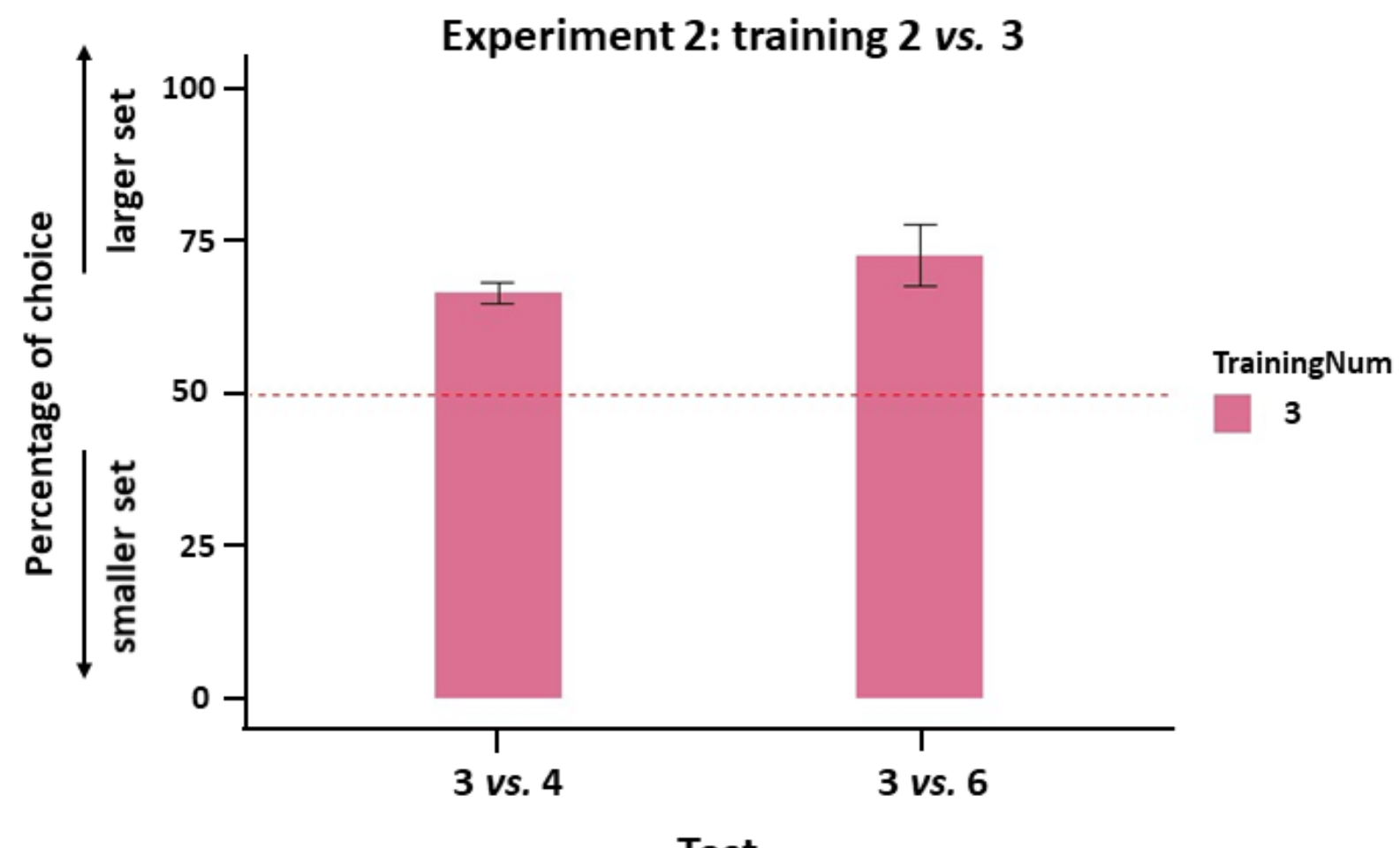
discrimination as found in other fish species $[26,58]$.

Figure 3. Percentage of choice for the larger/smaller set (mean \pm SEM) displayed by fish in the novel comparison tests for the group trained to select the larger (3) set.

228 The stimuli used in our Experiments were visual collections of black dots differing in numerosity. As described in the method section, for each numerical comparison, the

230 physical properties of each array were equalized for the geometry (radius, area and 231 perimeter) and spatial disposition (inter-distance (density) and convex-hull; see Figure 
232 1). Since we are dealing with images, each figure could also be described in terms of 233 spatial frequency. Spatial frequency can be thought of as the number of repeating 234 elements in a pattern per unit distance, and it is mathematically described by the Fourier 235 transform theory. No control was applied to the spatial frequency of our stimuli. Thus, in 236 order to check whether spatial frequency could influence archerfish choice, we 237 calculated spatial frequency variation across all different numerosities and control 238 conditions (see Methods section). Within each numerical test comparison, different 239 spatial frequencies were found (see Figure 4). The different constraints applied to the 240 stimuli (control of the area, perimeter or elements radius) showed to influence differently 241 the spatial frequency between the two numerosities. In detail, when the elements' radius was fixed between the two numerical arrays, the total power of the spatial frequency was higher in the smaller group than in the larger one, while the opposite was found in

244 the groups in which the overall perimeter was balanced (total power higher in the more 245 numerous group). Interestingly, this trend was maintained in all the numerical 246 comparisons used, irrespective of the number of elements to be compared.

To investigate the influence of spatial frequency in the numerical task, we analyzed whether a correlation between the performance accuracy and the spatial 249 frequency was apparent, for all possible control configurations (see Methods section). 250 Results are reported in Figure 4, showing no correlations between any comparison (test 2512 vs. 3: $r(4)=-0.17, p=0.83$; test 3 vs. $4: r(4)=0.15, p=0.77$; test 3 vs. $6: r(4)=-0.35$, $252 \mathrm{p}=0.50$; test 5 vs. 8: $r(4)=-0.08, \mathrm{p}=0.88$; test 6 vs. $9: \mathrm{r}(4)=-0.42, \mathrm{p}=0.41$.

253 These data strongly suggest that the spatial frequency was not influencing archerfish 254 performance in the numerical task. 
Spatial Frequency

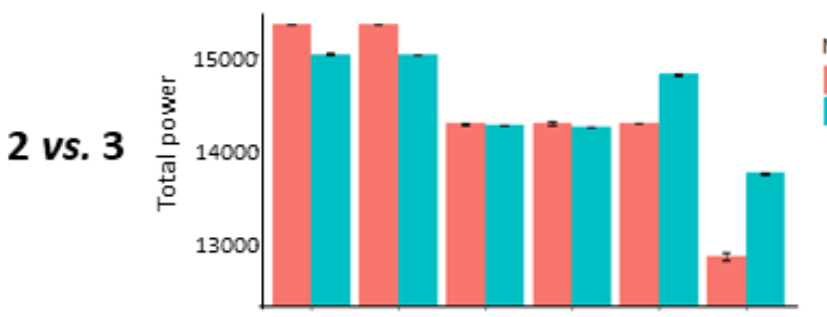

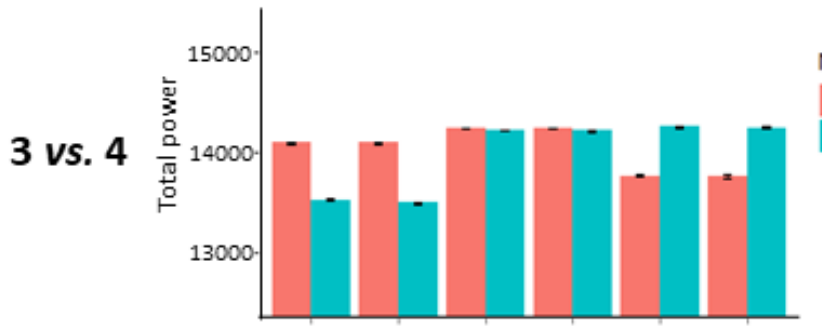

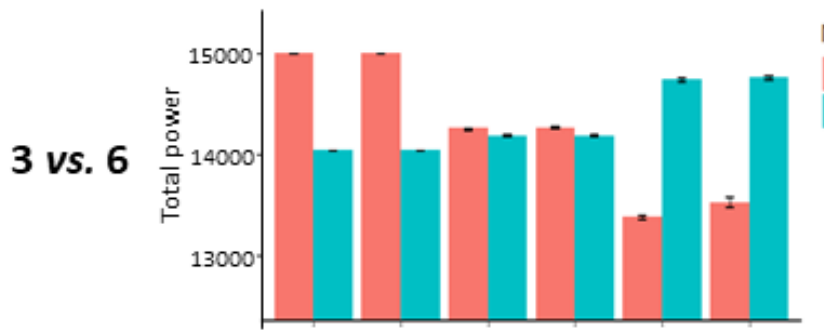
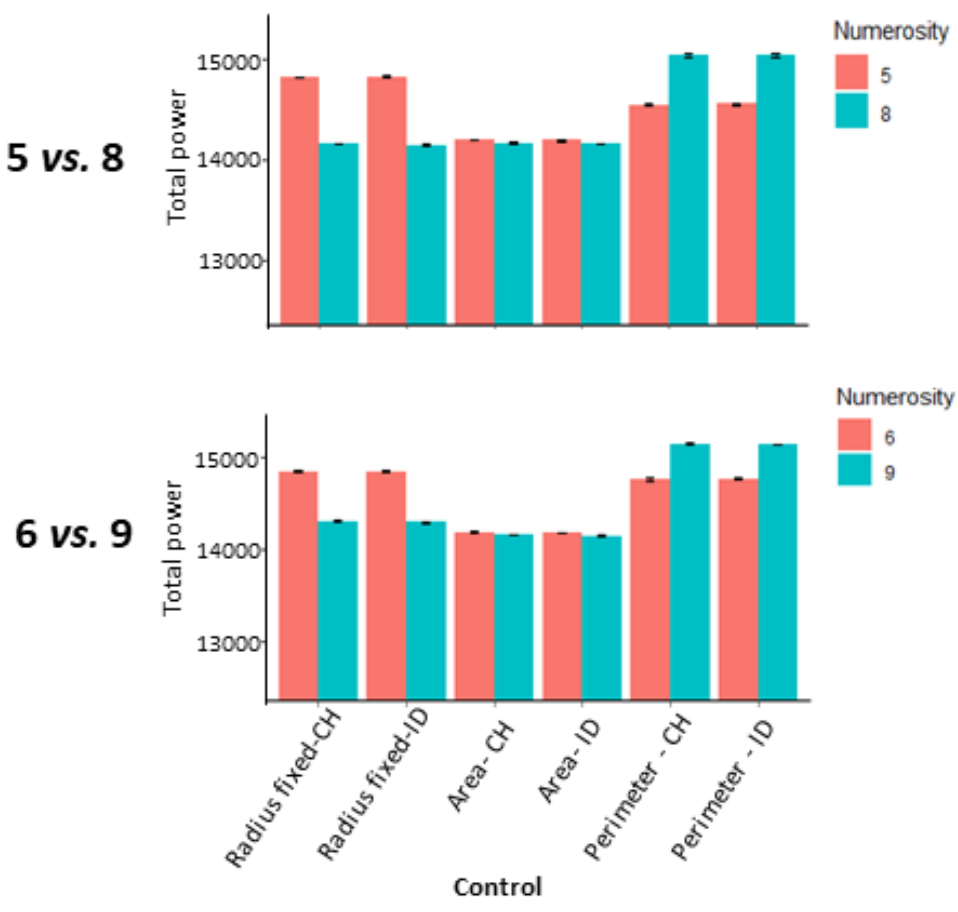

\section{Correlation}
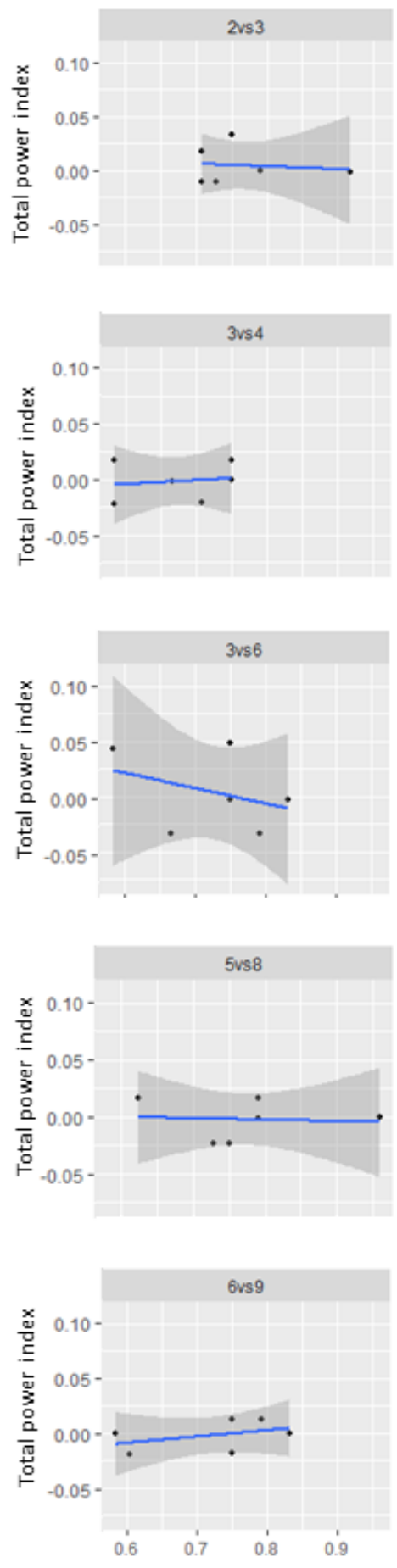

Choice for relative number 
Figure 4. The histograms (on the left) show the spatial frequency (Total power) for each numerical comparison among the different control groups (non-numerical variables control). The different constraints applied to the stimuli (control of the area, perimeter or elements radius) showed to influence the spatial frequency between the two compared numerosities. The regression lines (on the right) show the correlation between fish' performance accuracy (choice for the relative numerosity) and the spatial frequency (total power index between the two total power values), for all numerical comparisons.

\section{DISCUSSION}

Overall, our results showed that when trained to select a specific group of elements between two numerical arrays, archerfish spontaneously generalize at test to novel numerical comparison according to a relative numerical rule (select the largest/smallest) rather than an absolute numerical rule (select the specific number of items). These findings are in agreement with previous results from other fish species and humans $[55,56]$, while differing with respect to bees [57].

Interestingly, archerfish uses a general relative judgement even when trained to discriminate between numerosities that belong to different systems, namely small and large numerosities (for a review see [61]). In Experiment 1, archerfish were trained with a 3 vs. 6 contrast and then observed in test conditions with a 2 vs. 3, 6 vs .9 and 5 vs. 8 comparison. In all the tests, archerfish showed to spontaneously use a general relative rule. In Experiment 2, subjects' performance was observed in a numerical discrimination involving at training only small numerosities (i.e., 2 vs. 3). Once again, at test, fish followed the relative rule, selecting the largest group in the test comparisons 3 vs. 4 and 3 vs. 6 , thus ignoring the absolute number of elements (3). 
Taken together, our results support the hypothesis of a unique system for

282

283

284

285

286

287

288

289

290

291

292

293 294 applied to [62].

295

296

297

298

299

300

301

302 nest [29]. representing numerosities in archerfish, working both for small and large numbers, obeying the ANS. Evidence from other fish species supports this claim $[26,58]$.

The reason for which archerfish primarily rely on the relative information of numerical groups may have ecological reasons, being more adaptive in a natural environment that constantly require numerical/quantity judgement. Selecting the largest social group of companions or the largest food patch are easy rules that can be more efficient than using an absolute rule. Moreover, the use of relative information may be less cognitively expensive (in terms of memory load) than the absolute one, since it does not require storing the information about the precise number of elements: the discrimination could work on a simple relative comparison between numerosities, guided by the numerical ratio between the two. Nevertheless, the engagement of relative rules requires a good level of abstraction and the creation of a general rule to be

In fish, the use of an absolute rule may not be as convenient as the relative one, given that in most ecological contests there is not a specific optimal amount of food, partners or companions. However, this seems not to be the case for species such as bees, which showed instead a spontaneous use of absolute numerical information, suggesting that this rule may be more informative and useful in their ecological environment. Similar evidence has been found in spiders, that, in a natural predatory strategy context, settle their attack based on the specific number of conspecifics at the 
Note, however, that the spontaneous use of a relative or absolute rule does not imply that animals are unable to use both. Vertebrates can be trained to learn a specific number of items in a set if forced to do it $[6,56,63,64]$. Similarly, bees can be trained to 306 the numerical concepts of "greater than" or "smaller than" [47]. The spontaneous 307 engagement of one of the two criteria is therefore justified probably by a combination of 308 natural constraints and/or less cognitive demand motivations that better fit for the individuals' fitness in their particular niches of adaptation.

Lastly, with respect to the main question of our paper, the results showed that 311 archerfish are capable of abstract numerical discrimination, not influenced by other 312 continuous physical variables. We tested archerfish with numerical arrays well 313 controlled for all the possible non-numerical variables (e.g., total area, perimeter, inter314 distance (density), convex hull), thus ensuring that the discrimination made by the 315 animals was based on purely numerical information. The results of the statistical analyses showed no influence whatsoever of the different control conditions on the fish

317 choices. Moreover, we showed that even the different spatial frequencies of the stimuli were not influential on archerfish performance. The total power of the spatial frequency has been described in the literature to positively increase with numerosity [48]; however, 320 in our stimuli, the different geometrical constraints showed that it can be reversed as 321 well. Moreover, elements area and perimeter seem to play a crucial role in the 322 distribution of the spatial frequencies' energy with respect to the elements disposition 323 (inter-distance and sparsity). All our analyses suggested that the amplitude component 324 of the spatial frequency was not influencing archerfish numerical evaluation during our experiments. 
Note, however, that in all studies carried out so far (including our own analysis), the focus was on the amplitude of the spatial frequency as the main component, which provides information on the alternation rate of different elements in the image. It is likely

329 that a more specific role on computation of numerosity is played by the spatial 330 frequency phase component (related to elements' spatial coherence and 331 distribution) which directly relates to figure-ground aggregation and unity formation.

In conclusion, our results provide clear evidence that under conditions of strict control of continuous physical variables archerfish can encode an abstract concept of number to support relative numerical judgement for both small and large numerosities.

\section{MATERIALS AND METHODS}

SUBJECTS AND REARING CONDITIONS

337 Sixteen adult archerfish, Toxotex jaculatrix (fish size ranged between 8 and $10 \mathrm{~cm}$ in 338 length) were provided by a local commercial supplier (“Acquario G di Segatta Stefano").

339 Four animals were excluded because they did not show any consistent motivation in 340 hitting the screen. A group of fish $(\mathrm{N}=8)$ took part in Experiment 1, while a second group $341(\mathrm{~N}=4)$ took part in Experiment 2. All fish were housed in large aquariums $(100 \times 40 \times 40$ $342 \mathrm{~cm}$ ) in groups of 10 individuals. Prior to the experiment, each archerfish was moved into 343 individual aquaria $(40 \times 30 \times 50 \mathrm{~cm})$ filled with freshwater maintained at $25^{\circ} \mathrm{C}$ and 344 enriched with gravel and a shelter. Water quality was kept by suitable filters (Sera fil 345 60). The system was illuminated under a 10:14 light /dark cycle (Sylvania luxline plus 346 F36W/840 cool white). Fish were fed with food pellets (Hikari cichlid gold baby pellet). 
APPARATUS

348 Both the apparatus and the training method were set up based on previous studies

349 conducted with archerfish on visual discrimination tasks (i.e., [52,53]). Each experimental tank consisted of a rectangular aquarium with a monitor screen located

351 above it (20", DELL 2009Wt), held at $30 \mathrm{~cm}$ from the water level (Figure 5a). Each tank was surrounded by white opaque panels to ensure that the fish was not distracted by external cues. Each tank was raised $8 \mathrm{~cm}$ off the table thanks to lateral supports,

354 allowing the positioning of a video camera under the centre of the pavement's tank to record a bottom view of the fish and the screen (see video example in the supplementary materials).

a
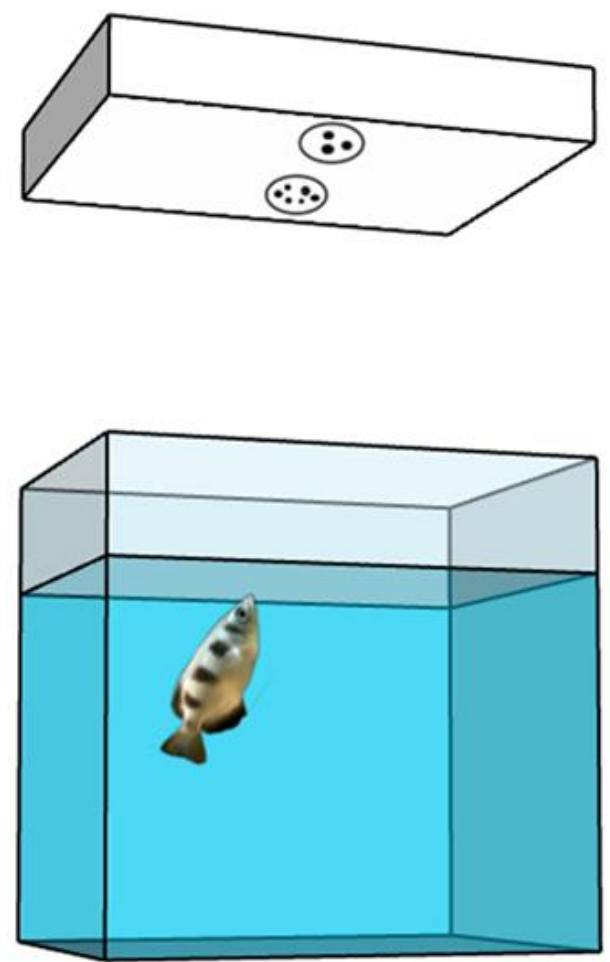

b

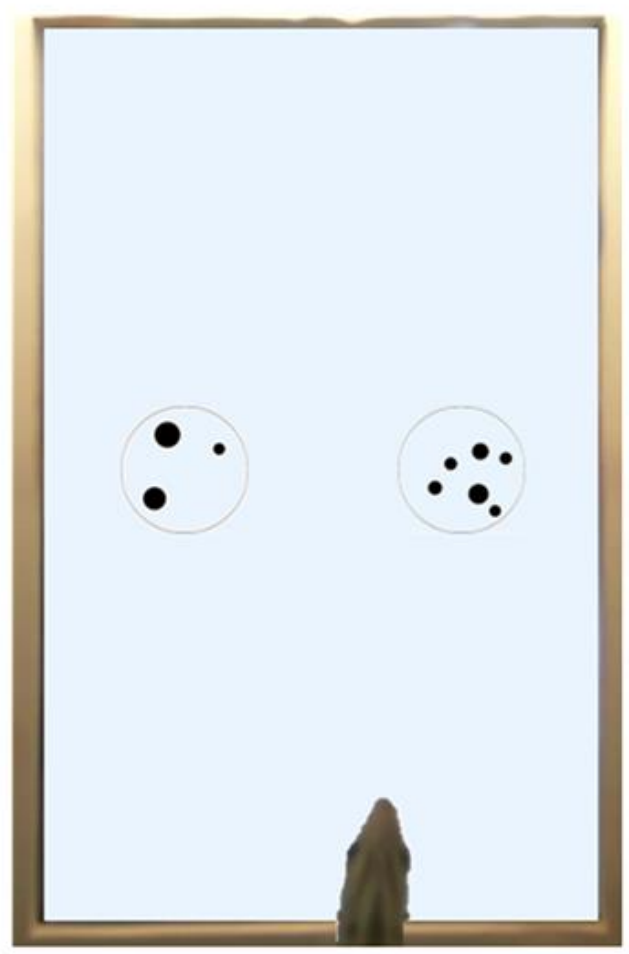


Figure 5. a) Schematic representation of the experimental setup; b) Bottom view of the tank from the camera placed below the tank's pavement.

STIMULI

363 The stimuli presented in the training phase consisted of groups of black dots confined

364 into a black outline circle (6 cm diameter). The dots size was ranging between 3 and 12 $365 \mathrm{~mm}$, and the visual angle was in the range $0.43^{\circ}$ and $1.72^{\circ}$, which has been proven to 366 be well perceived by archerfish [54]. In every trial, a couple of stimuli was 367 simultaneously presented in the centre of the screen (horizontally aligned to the shortest 368 monitor's side, see Figure 5b). All the stimuli were created using the software GeNEsIS 369 [49], a Matlab program that allows to create numerical collections of stimuli controlled 370 for several non-numerical magnitudes. Given that it is mathematically impossible to

371 balance all the non-numerical magnitudes simultaneously in two different numerical 372 groups, different sets of stimuli were created for each numerosity, controlling for some 373 visual physical property; all the possible properties were covered across the different

374 sets during a session (see Figure 1 for a view of all the combinations applied in a 375 session). Pictures from each set were randomly presented, making the numerical 376 information the only reliable cue to differentiate the two stimuli across all the various 377 trials.

378 GENERAL PROCEDURE

379 Pre-training phase

380 Before starting the experiment, fish underwent a pre-training phase in which they were 381 gradually habituated to spit (hit with a jet of water) at the training stimulus on the screen. 382 This was accomplished throughout a shaping procedure to facilitate the task. The 
383 silhouette of an insect was initially presented, inducing the fish reaction to spit at the 384 prey; once hit, fish were rewarded with a food pellet. The insect was gradually replaced by a black dot and finally with the effective training stimulus. Once the fish accomplished all these stages, the training phase was initiated.

Training phase

388 Fish were trained to spit at the correct target presented on the monitor above the tank.

389 The stimuli to discriminate consisted of two groups of dots with different numerosity.

390 Every trial started with the appearance of a blinking black square $(1.6 \mathrm{~cm}$, three blinks 391 of 100 milliseconds) at the centre of the screen to catch the fish's attention towards the 392 screen. Then, the two training stimuli were displayed one next to the other (distance 7 $393 \mathrm{~cm}$ ) on the two sides of the monitor. Only one of the two numerosities was rewarded 394 with a food pellet when hit, while the choice for the incorrect stimulus caused the stop of the trial, which in every case, in absence of choice, was stopped after 5 minutes. At the 396 end of each trial, the screen was cleaned from the water drops and a new trial started.

397 In the first training session only, a corrective method was applied: the stimuli remained 398 on the screen until the subject selected the correct target, even if the incorrect stimulus was hit, allowing the fish to correct its choice.

Fish were trained with daily sessions of 48 trials, in which continuous physical 401 variables were controlled and changed according to the scheme reported in Figure 1, 402 and the position of the target stimulus on the screen (right-left) was randomized. Fish 403 generally responded $70 \%$ to $100 \%$ of the trials. The learning phase was considered 404 completed when the fish reached a learning criterion of at least $75 \%$ of correct choices 
405 for two consecutive days (binomial test: $p<0.01$ ), allowing the fish to take part in the 406 test phase.

407

408 Test phase

409 Generally, each test condition consisted of the presentation of a couple of stimuli with a

410 novel numerical comparison, aiming to see if the numerosity target learned in the

411 training phase was represented as a relative or an absolute numerical information. Each

412 test was composed of 24 probe trials not rewarded, divided into three testing days of 8

413 trials. In each test session, the 8 test trials were shuffled and interspersed with

414 rewarded recall training trials (32 recall in total), to maintain the fish motivation high

415 during the whole test duration. The order of the tests was randomized among the fish to 416 exclude that the performance could be influenced by their order. At the end of each test,

417 the fish underwent a complete daily session of retraining to further exclude potential 418 interference among the tests.

419 Statistical analyses and data analysis

420 Data were analyzed using $R$ software (R-4.1.0). In Experiment 1, an independent t-test

421 was used to compare the number of trials to reach the criterion between the two groups

422 at training. At test (Exps. 1 and 2), choices for the relative numerosity were analyzed

423 using a generalized linear mixed model fit by maximum likelihood (Laplace 424 Approximation), binomial GLMM with a logit link. A binomial test was used to compare 425 the distribution of the choices for the relative and absolute numerosities.

426 To obtain an estimate of the spatial frequency we adopted an approach already 427 performed in other studies $[48,59,60]$ : the fast Fourier transform of our images was 
428 calculated, a radial average of the signal amplitude in the frequency domain was 429 performed, and lastly, all the frequency contributions of its power spectrum were

430 summed up. In this way, a value related to the total energy of each frequency

431 component inside a given image is obtained.

432 To investigate the influence of spatial frequency in the numerical task, we analyzed

433 whether a correlation between the performance accuracy (choice for the relative 434 numerosity) and the spatial frequency (normalized total power difference between the 435 two compared numerosities) was apparent, for all possible control configurations. To 436 compare two numerosities we reported a normalized difference (total power index)

437 between the two total power values (difference between the total power of the biggest 438 numerosity and the smallest, divided by their sum). All the frequency calculations were 439 performed with a custom script in Matlab, while the statistical comparisons were 440 calculated in R. For each of them a Pearson's correlation coefficient was calculated 441 comparing the choice for the relative numerosity and the normalized difference between 442 numerosities (as explained above). 


\section{Ethical regulation}

445 The present research was carried out at the Animal Cognition and Neuroscience

446 Laboratory (ACN Lab) of the CIMeC (Center for Mind/Brain Sciences), at the University

447 of Trento (Italy). All husbandry and experimental procedures complied with European

448 Legislation for the Protection of Animals used for Scientific Purposes (Directive 449 2010/63/EU) and were approved by the Scientific Committee on Animal Health and

450 Animal Welfare (Organismo Preposto al Benessere Animale, OPBA) of the University of

451 Trento and by the Italian Ministry of Health (Protocol n. 932/2020-PR).

\section{Competing interests}

453 We declare we have no competing interests.

\section{$454 \quad$ Funding}

455 This project has received funding from the European Research Council (ERC) under the 456 European Union's Horizon 2020 research and innovation program (grant agreement No 457833504 SPANUMBRA to G.V.) and Progetti di Rilevante Interesse Nazionale (PRIN 4582017 ERC-SH4-A 2017PSRHPZ to G.V.)

\section{Authors' contribution}

460 D.P. and G.V conceived the study. D.P. G.V and M.Z. designed the experiment. D.P. 461 performed the experiments. All authors interpreted the data and contributed to the 462 manuscript writing. 


\section{BIBLIOGRAPHY}

465

466

467

468

469

470

471

472

473

474

475

476

477

478

479

480

481

482

483

484

485

486

487

488

489

490

491

492

493

494

495

496

497

498

499

1. Nieder A. 2020 The Adaptive Value of Numerical Competence. Trends Ecol. Evol. 35, 605-617. (doi:10.1016/j.tree.2020.02.009)

2. Agrillo C, Bisazza A. 2014 Spontaneous versus trained numerical abilities. A comparison between the two main tools to study numerical competence in nonhuman animals. J. Neurosci. Methods 234, 82-91. (doi:10.1016/j.jneumeth.2014.04.027)

3. Anderson US, Stoinski TS, Marr MJ, Smith AD, Bloomsmith MA, Maple TL. 2005 Relative numerousness judgment and summation in young and old Western lowland gorillas. J. Comp. Psychol. 119, 285-295. (doi:10.1037/07357036.119.3.285)

4. Beran MJ, Evans TA, Leighty KA, Harris EH, Rice D. 2008 Summation and quantity judgments of sequentially presented sets by capuchin monkeys (Cebus apella). Am. J. Primatol. 70, 191-194. (doi:10.1002/ajp.20474)

5. Beran MJ, Beran MM. 2004 Chimpanzees Remember the Results of One-by-One Addition of Food Items to Sets Over Extended Time Periods. Psychol. Sci. 15, 94-99. (doi:10.1111/j.0963-7214.2004.01502004.x)

6. Cantlon JF, Brannon EM. 2007 How much does number matter to a monkey (Macaca mulatta)? J. Exp. Psychol. Anim. Behav. Process. 33, 32-41. (doi:10.1037/0097-7403.33.1.32)

7. Smith BR, Piel AK, Candland DK. 2003 Numerity of a Socially Housed Hamadryas Baboon (Papio hamadryas) and a Socially Housed Squirrel Monkey (Saimiri sciureus). J. Comp. Psychol. 117, 217-225. (doi:10.1037/07357036.117.2.217)

8. Abramson JZ, Hernández-Lloreda V, Call J, Colmenares F. 2013 Relative quantity judgments in the beluga whale (Delphinapterus leucas) and the bottlenose dolphin (Tursiops truncatus). Behav. Processes 96, 11-19. (doi:10.1016/j.beproc.2013.02.006)

9. Benson-Amram S, Heinen VK, Dryer SL, Holekamp KE. 2011 Numerical assessment and individual call discrimination by wild spotted hyaenas, Crocuta crocuta. Anim. Behav. 82, 743-752. (doi:10.1016/j.anbehav.2011.07.004)

10. McComb K, Packer C, Pusey A. 1994 Roaring and numerical assessment in contests between groups of female lions, Panthera leo. Anim. Behav. 47, 379387. (doi:10.1006/anbe.1994.1052)

11. Perdue BM, Talbot CF, Stone AM, Beran MJ. 2012 Putting the elephant back in the herd: Elephant relative quantity judgments match those of other species. 
Anim. Cogn. 15, 955-961. (doi:10.1007/s10071-012-0521-y)

12. Vonk J, Beran MJ. 2012 Bears 'count' too: Quantity estimation and comparison in black bears, Ursus americanus. Anim. Behav. 84, 231-238. (doi:10.1016/j.anbehav.2012.05.001)

13. West RE, Young RJ. 2002 Do domestic dogs show any evidence of being able to count? Anim. Cogn. 5, 183-186. (doi:10.1007/s10071-002-0140-0)

14. Bogale BA, Aoyama M, Sugita S. 2014 Spontaneous discrimination of food quantities in the jungle crow, Corvus macrorhynchos. Anim. Behav. 94, 73-78. (doi:10.1016/j.anbehav.2014.05.012)

15. Ditz HM, Nieder A. 2016 Numerosity representations in crows obey the WeberFechner law. Proc. R. Soc. B Biol. Sci. 283. (doi:10.1098/rspb.2016.0083)

16. Garland A, Low J, Burns KC. 2012 Large quantity discrimination by North Island robins (Petroica longipes). Anim. Cogn. 15, 1129-1140. (doi:10.1007/s10071012-0537-3)

17. Pepperberg IM. 2006 Grey parrot (Psittacus erithacus) numerical abilities: Addition and further experiments on a zero-like concept. J. Comp. Psychol. 120, 1-11. (doi:10.1037/0735-7036.120.1.1)

18. Rugani R, Vallortigara G, Regolin L. 2013 Numerical Abstraction in Young Domestic Chicks (Gallus gallus). PLoS One 8, 4-9. (doi:10.1371/journal.pone.0065262)

19. Scarf D, Colombo M. 2011 Knowledge of the Ordinal Position of List Items in Pigeons. J. Exp. Psychol. Anim. Behav. Process. 37, 483-487. (doi:10.1037/a0023695)

20. Krusche P, Uller C, Dicke U. 2010 Quantity discrimination in salamanders. J. Exp. Biol. 213, 1822-1828. (doi:10.1242/jeb.039297)

21. Stancher G, Rugani R, Regolin L, Vallortigara G. 2015 Numerical discrimination by frogs (Bombina orientalis). Anim. Cogn. 18, 219-229. (doi:10.1007/s10071014-0791-7)

22. Gazzola A, Vallortigara G, Pellitteri-Rosa D. 2018 Continuous and discrete quantity discrimination in tortoises. Biol. Lett. 14, 1-4. (doi:10.1098/rsbl.2018.0649)

23. Petrazzini MEM, Bertolucci C, Foà A. 2018 Quantity discrimination in Trained lizards (Podarcis sicula). Front. Psychol. 9, 1-6. (doi:10.3389/fpsyg.2018.00274)

24. Gómez-Laplaza LM, Díaz-Sotelo E, Gerlai R. 2018 Quantity discrimination in angelfish, Pterophyllum scalare: a novel approach with food as the discriminant. 
Anim. Behav. 142, 19-30. (doi:10.1016/j.anbehav.2018.06.001)

25. Potrich D, Rugani R, Sovrano VA, Regolin L, Vallortigara G. 2019 Use of numerical and spatial information in ordinal counting by zebrafish. Sci. Rep. 9:18323. (doi:10.1038/s41598-019-54740-8)

26. Stancher G, Sovrano VA, Potrich D, Vallortigara G. 2013 Discrimination of small quantities by fish (redtail splitfin, Xenotoca eiseni). Anim. Cogn. 16, 307-312. (doi:10.1007/s10071-012-0590-y)

27. Dacke M, Srinivasan M V. 2008 Evidence for counting in insects. Anim. Cogn. 11, 683-689. (doi:10.1007/s10071-008-0159-y)

28. Gross HJ, Pahl M, Si A, Zhu H, Tautz J, Zhang S. 2009 Number-based visual generalisation in the honeybee. PLoS One 4, e4263. (doi:10.1371/journal.pone.0004263)

29. Nelson XJ, Jackson RR. 2012 The role of numerical competence in a specialized predatory strategy of an araneophagic spider. Anim. Cogn. 15, 699-710. (doi:10.1007/s10071-012-0498-6)

30. Rodríguez RL, Briceño RD, Briceño-Aguilar E, Höbel G. 2015 Nephila clavipes spiders (Araneae: Nephilidae) keep track of captured prey counts: testing for a sense of numerosity in an orb-weaver. Anim. Cogn. 18, 307-314. (doi:10.1007/s10071-014-0801-9)

31. Vallortigara G. 2017 An animal's sense of number. In The nature and development of mathematics. Cross disciplinary perspective on cognition, learning and culture (ed A Adams, J. W., Barmby, P. \& Mesoudi), pp. 43-65. Routledge, New York.

32. Bortot M, Regolin L, Vallortigara G. 2021 A sense of number in invertebrates. Biochem. Biophys. Res. Commun. 564, 37-42. (doi:10.1016/j.bbrc.2020.11.039)

33. Butterworth Brian. 1999 The mathematical brain. Macmillan. London.

34. Nieder A, Dehaene S. 2009 Representation of number in the brain. Annu. Rev. Neurosci. 32, 185-208. (doi:10.1146/annurev.neuro.051508.135550)

35. Trick LM, Pylyshyn ZW. 1994 Why are small and large numbers enumerated differently? A limited-capacity preattentive stage in vision. Psychol. Rev. 101, 80102. (doi:10.1037/0033-295X.101.1.80)

36. Vallortigara G. 2014 Foundations of number and space representations in precocial species. In Evolutionary origins and early development of number processing (ed K Geary, D. C., Bearch, D. B. \& Mann Koepke), pp. 35-66. New York: Elsevier. 
37. Kutter EF, Bostroem J, Elger CE, Mormann F, Nieder A. 2018 Single Neurons in the Human Brain Encode Numbers. Neuron 100, 753-761.e4. (doi:10.1016/j.neuron.2018.08.036)

38. Piazza M, Izard V, Pinel P, Le Bihan D, Dehaene S. 2004 Tuning curves for approximate numerosity in the human intraparietal sulcus. Neuron 44, 547-555. (doi:10.1016/j.neuron.2004.10.014)

39. Nieder A, Freedman DJ, Miller EK. 2002 Representation of the quantity of visual items in the primate prefrontal cortex. Science 297, 1708-1711. (doi:10.1126/science.1072493)

40. Nieder A, Merten K. 2007 A labeled-line code for small and large numerosities in the monkey prefrontal cortex. J. Neurosci. 27, 5986-5993. (doi:10.1523/JNEUROSCI.1056-07.2007)

41. Ditz HM, Nieder A. 2015 Neurons selective to the number of visual items in the corvid songbird endbrain. Proc. Natl. Acad. Sci. U. S. A. 112, 7827-7832. (doi:10.1073/pnas.1504245112)

42. Messina A, Potrich D, Schiona I, Sovrano VA, Fraser SE, Brennan $\mathrm{CH}$, Vallortigara G. 2020 Response to change in the number of visual stimuli in zebrafish:A behavioural and molecular study. Sci. Rep. 10:5769. (doi:10.1038/s41598-020-62608-5)

43. Messina A, Potrich D, Schiona I, Sovrano VA, Fraser SE, Brennan $\mathrm{CH}$, Vallortigara G. 2021 Neurons in the Dorso-Central Division of Zebrafish Pallium Respond to Change in Visual Numerosity. Cereb. Cortex 00, 1-11. (doi:10.1093/cercor/bhab218)

44. Nieder A. 2021 The Evolutionary History of Brains for Numbers. Trends Cogn. Sci. 25, 608-621. (doi:10.1016/j.tics.2021.03.012)

45. Vallortigara G. 2021 Born Knowing: Imprinting and the Origins of Knowledge. Cambridge, Ma: MIT PRESS.

46. Leibovich T, Katzin N, Harel M, Henik A. 2017 From 'sense of number' to 'sense of magnitude': The role of continuous magnitudes in numerical cognition. Behav. Brain Sci. 40. (doi:10.1017/S0140525X16000960)

47. Howard SR, Avarguès-Weber A, Garcia JE, Greentree AD, Dyer AG. 2018 Numerical ordering of zero in honey bees. Science 360, 1124-1126. (doi:10.1126/science.aar4975)

48. Maboudi H Di et al. 2021 Non-numerical strategies used by bees to solve numerical cognition tasks. Proc. R. Soc. B Biol. Sci. 288, 20202711. (doi:10.1098/rspb.2020.2711) 
621

622

623

624

625

626

627

628

629

630

631

632

633

634

635

636

637

638

639

640

641

49. Zanon M, Potrich D, Bortot M, Vallortigara G. 2021 Towards a standardization of non-symbolic numerical experiments: GeNEsIS, a flexible and user-friendly tool to generate controlled stimuli. Behav. Res. Methods (doi:10.3758/s13428-02101580-y)

50. Newport C, Schuster S. 2020 Archerfish vision: Visual challenges faced by a predator with a unique hunting technique. Semin. Cell Dev. Biol. 106, 53-60. (doi:10.1016/j.semcdb.2020.05.017)

51. Leibovich-Raveh T, Raveh A, Vilker D, Gabay S. 2021 Magnitude integration in the Archerfish. Sci. Rep. 11, 1-11. (doi:10.1038/s41598-021-94956-1)

52. Karoubi N, Leibovich T, Segev R. 2017 Symbol-value association and discrimination in the archerfish. PLoS One 12, 1-16. (doi:10.1371/journal.pone.0174044)

53. Newport C, Wallis G, Temple SE, Siebeck UE. 2013 Complex, context-dependent decision strategies of archerfish, Toxotes chatareus. Anim. Behav. 86, 12651274. (doi:10.1016/j.anbehav.2013.09.031)

54. Ben-Simon A, Ben-Shahar O, Vasserman G, Ben-Tov M, Segev R. 2012 Visual acuity in the archerfish: Behavior, anatomy, and neurophysiology. J. Vis. 12, 119. (doi:10.1167/12.12.18)

55. Miletto Petrazzini ME, Agrillo C, Izard V, Bisazza A. 2016 Do humans (Homo sapiens) and fish (Pterophyllum scalare) make similar numerosity judgments. $J$. Comp. Psychol. 130, 380-390. (doi:10.1037/com0000045)

56. Miletto Petrazzini ME, Agrillo C, Izard V, Bisazza A. 2015 Relative versus absolute numerical representation in fish: Can guppies represent "fourness"? Anim. Cogn. 18, 1007-1017. (doi:10.1007/s10071-015-0868-y)

57. Bortot M, Agrillo C, Avarguès-Weber A, Bisazza A, Petrazzini MEM, Giurfa M. 2019 Honeybees use absolute rather than relative numerosity in number discrimination. Biol. Lett. 15, 5-9. (doi:10.1098/rsbl.2019.0138)

58. Potrich D, Sovrano VA, Stancher G, Vallortigara G. 2015 Quantity discrimination by zebrafish (Danio rerio). J. Comp. Psychol. 129, 388-393. (doi:10.1037/com0000012)

59. Adriano A, Girelli L, Rinaldi L. 2021 The ratio effect in visual numerosity comparisons is preserved despite spatial frequency equalisation. Vision Res. 183, 41-52. (doi:10.1016/j.visres.2021.01.011)

60. Felisatti A, Laubrock J, Shaki S, Fischer MH. 2020 A biological foundation for spatial-numerical associations: the brain's asymmetric frequency tuning. Ann. N. Y. Acad. Sci. 1477, 44-53. (doi:10.1111/nyas.14418) 
642 61. Hyde DC. 2011 Two systems of non-symbolic numerical cognition. Front. Hum.

$643 \quad$ Neurosci. 5, 1-8. (doi:10.3389/fnhum.2011.00150)

644 62. Pepperberg IM, Brezinsky M V. 1991 Acquisition of a relative class concept by an

645

646 African gray parrot (Psittacus erithacus): discriminations based on relative size. $J$. Comp. Psychol. 105, 286-294. (doi:10.1037/0735-7036.105.3.286)

647

63. Pepperberg IM. 1994 Numerical competence in an African gray parrot (Psittacus erithacus). J. Comp. Psychol. 108, 36-44. (doi:10.1037/0735-7036.108.1.36)

649 64. Smirnova AA, Lazareva OF, Zorina ZA. 2000 Use of Number By Crows:

650 Investigation By Matching and Oddity Learning. J. Exp. Anal. Behav. 73, 163-

651 176. (doi:10.1901/jeab.2000.73-163) 